\title{
Obstructive sleep apnea: past, present and future
}

\author{
Gilbert Berdine MD
}

With these words, "...and on the box sat a fat and red-faced boy, in a state of somnolency, ..." Charles Dickens introduced the reader of The Pickwick Papers to the memorable character known as Joe The Fat Boy. ${ }^{1}$ This description led Burwell et al to attach the label Pickwickian Syndrome to their description of a patient with obesity and central alveolar hypoventilation. ${ }^{2}$ Their case report began with quotes from Dickens, continued with measurements of respiratory function before and after weight loss, and concluded that "there is a critical degree of obesity at which ventilatory insufficiency appears." Of note, the patient had a weight of $121.4 \mathrm{~kg}$ which was extraordinary at the time but is commonplace today. It would be 10 years before it was recognized that Joe the Fat Boy (and likely Burwell's case report) suffered from Obstructive Sleep Apnea rather than some form of central alveolar hypoventilation.

Gastaut et al described a case very similar to Joe the Fat Boy and performed polysomnography to determine the etiology of the problem. ${ }^{3}$ Their patient "was a man of average height $(172 \mathrm{~cm})$, weighing 125 $\mathrm{kg}$. The face was bloated and congested but showed no cyanosis. In the course of the interview and the examination the patient fell asleep spontaneously." Their polysomnogram demonstrated a repeated pattern of diminished or absent airflow despite persistent contraction of the diaphragm. The pattern illustrated in their paper has become the classic graphic pattern of obstructive apnea. It should again be noted that the weight of their patient would be hardly remarkable today.

Corresponding author: Gilbert Berdine MD Contact Information: Gilbert.Berdine@ttuhsc.edu DOI: 10.12746/swrccc 2016.0415.203
The next breakthrough in understanding was a demonstration in 1978 by Remmers et al that periods of obstructive apnea coincided with diminished or absent EMG activity in the genioglossus. ${ }^{4}$ This led to the first demonstration that continuous positive airway pressure (CPAP) was effective as treatment for obstructive sleep apnea. ${ }^{5}$ The CPAP era was born. Of note, this study only had 5 patients, all of whom tolerated the nasal mask, and the levels of CPAP necessary to abolish apnea events were a very modest $4.5-10.0 \mathrm{cmH}_{2} \mathrm{O}$.

OSA offers a classic example of how government interference with the free market increases the cost of health care. Prior to 1981, there were no CPAP machines for sale, and the use of polysomnography was very limited. Government regulations limit what can be called a CPAP machine and what can be called a sleep test. Figure 1 illustrates how impairment to the supply of services raises the price of the service.

\section{Figure 1}

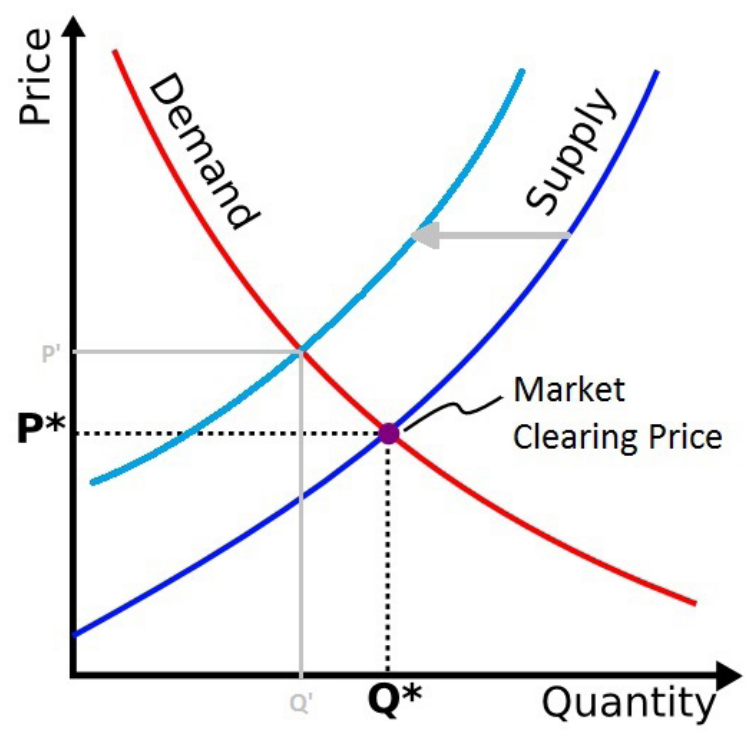


The supply curve in dark blue has a positive slope; the quantity offered for sale increases with price. The demand curve in red has a negative slope; the quantity bid for purchase decreases with price. The intersection of the two curves defines the price and quantity where the market clears, leaving neither unsatisfied buyers nor sellers. Regulations limit the available options and reduce the quantity offered for sale at each price. This is known as a shift of the supply curve to the left. The new curve for regulated supply intersects the demand curve at a lower quantity and higher price. The lower quantity is the intent of the regulation, usually touted as consumer protection. The higher price is the unintended side effect. This side effect of regulation cannot be avoided. Regulations are usually defended as necessary to protect the public from unscrupulous merchants. This is why toasters, computers, and cellphones are affordable to the average person while sleep testing and CPAP machines are not. Cellphones are certainly more complicated than CPAP machines yet cost much less.

Once regulation makes the service unaffordable by the average patient, subsidies are demanded by patients and government. Rather than solving the problem created by government regulation, the subsidies further increase the cost of delivering CPAP and sleep tests. This is illustrated by Figure 2 .

\section{Figure 2}

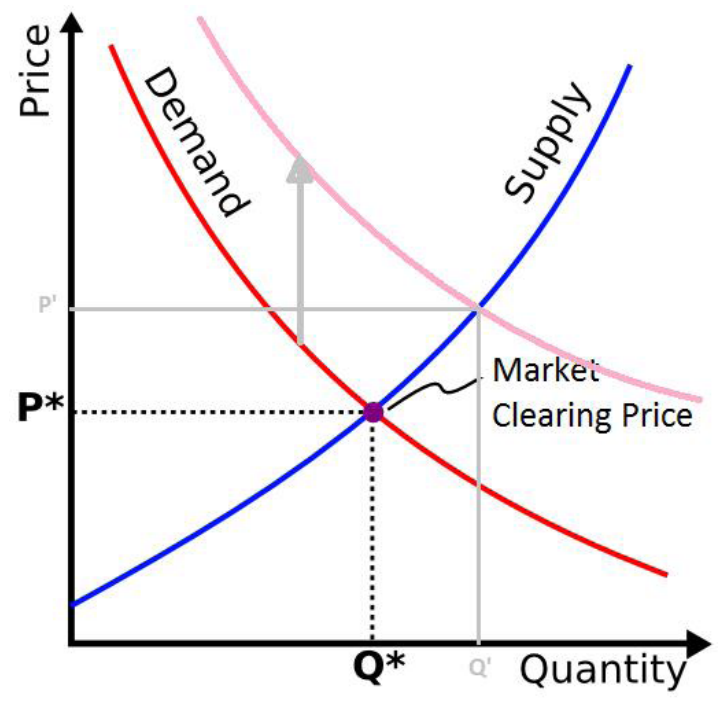

The subsidy makes the price appear less to the buyer. For any quantity, buyers will pay a higher price than they would pay without the subsidy. This shifts the demand curve up, though this is commonly called shifting the demand curve to the right. The new subsidized demand curve intersects the supply curve at a higher quantity and higher price. The higher quantity is the intent of the subsidy, but the higher price is the unavoidable unintended side effect. With the subsidy provided to anyone who "qualifies," the focus of work in OSA shifts away from providing a better product at a lower price (competitive innovation) and towards increasing the number of people deemed "qualified" for subsidy as much as possible (rent seeking). The ideal situation for the CPAP machine producer or sleep test provider is for the entire population to become "qualified" for the subsidy. Thus, one sees research associating OSA with other problems, such as hypertension and heart failure. The expansion of the number of people who are candidates for sleep testing leads to an increase in the demand for both sleep testing and CPAP therapy. The cost of health care rises.

The subsidy for sleep services led to a rapid expansion of the market for these services. From obscure beginnings around 1980, rapid growth in sleep testing and CPAP machines occurred. The Office of the Inspector General estimated that Medicare paid about $\$ 565$ million for sleep testing in $2011 .{ }^{6}$ Estimates of the total market for sleep services run into the tens of billions annually. ${ }^{7}$

The next phase of market distortion has two components, both related to the unintended price increase of the subsidy. If the subsidy is not offered to everyone, then there are two demand curves. There is one curve for buyers who do not qualify for the subsidy and another curve at a higher price for the subsidized buyer. It is well known that some patients who "qualify" for the subsidy do not continue using the CPAP machine. These patients have an arbitrage opportunity to sell machines they do not wish to use at a discount to patients who want the machine but do not qualify for the subsidy. Thus, we see the sale of CPAP machines on Craigslist. 
The second distortion is reaction by the payers against the rising cost of providing CPAP machines and sleep tests. The subsidies disconnect the consumers from the costs of the sleep tests and the CPAP machines, but the payers - government or insurers - have an interest in lowering the costs. Both groups of payers have an interest in disqualifying as many people as possible from the benefit of CPAP. Here is one example of a regulatory hurdle to qualify for CPAP therapy.

The patient must have a face to face evaluation with a physician of their choice. At this appointment there must be documentation of symptoms of OSA, a completed Epworth Sleepiness Scale, BMI (Body Mass Index), neck circumference, and a focused cardiopulmonary and upper airway system evaluation. This appointment with the physician must always proceed [sic] the baseline sleep study. ${ }^{8}$

On the surface, this regulation seems reasonable, but why must a patient have a neck circumference measurement since the use of CPAP will not depend on the result of that measurement? If, for whatever reason, the patient had a diagnostic sleep test demonstrating an $\mathrm{AHI}$ of 80 , why would CPAP therapy be denied simply because a neck circumference measurement and BMl calculation was not made prior to the sleep test? The Epworth Sleepiness Scale is a useful tool for screening patients, but it is not a reliable indicator of who actually suffers from OSA. The regulation becomes less about screening suitable candidates for CPAP and more about delaying a costly therapy or test. Other regulations require the use of a monitoring chip in the CPAP machine to document compliance with therapy. Unlike the CPAP Gestapo, providers of toasters and cellphones do not care how often their consumers use their products. Since the consumer is directly connected to the purchase price, the consumer can determine meaningful use by whatever criteria they choose. The subsidy for CPAP testing and equipment becomes necessary to afford therapy and inexorably leads to a maze of reg- ulatory burdens the patient must overcome to obtain therapy.

Another feature of the current landscape in sleep medicine is the changing nature of the patient. Contrast the success of the first clinical trial of CPAP using pressures $10 \mathrm{cmH}_{2} \mathrm{O}$ or less with the current use of pressures exceeding $20 \mathrm{cmH}_{2} \mathrm{O}$, the necessity of BiPAP, and the increasing failure of positive airway pressure to adequately treat the patient. It seems likely that increasing BMI is a culprit for these changes in patient outcomes.

The future of sleep medicine is murky. As part of government efforts to categorize who should qualify for subsidy, the government agencies have distilled all of the complex information within a diagnostic sleep test into a single number: the apnea-hypopnea index $(\mathrm{AHI})$. The determination of $\mathrm{AHI}$ does not require all of the information contained within a standard polysomnogram. The Home Sleep Test can determine an $\mathrm{AHI}$ at a small fraction of the cost of a laboratory sleep test. Nor does interpretation of a Home Sleep Test require years of specialized training with its attendant certificate. While there is no doubt that laboratory testing provides more information with greater accuracy, it is unclear that the increase in information and accuracy is worth the additional cost. United Healthcare will no longer approve laboratory sleep tests for the diagnosis of sleep apnea unless the patient has certain co-morbid conditions. ${ }^{9}$ It is ironic that Medicare initially required laboratory sleep testing for CPAP prescriptions to avoid fraudulent prescriptions. Medicare may eventually go full circle and require home sleep testing for CPAP prescriptions to avoid the costs of laboratory testing.

Consider this patient with OSA. The diagnostic sleep test demonstrated an $\mathrm{AHI}$ of 124. 


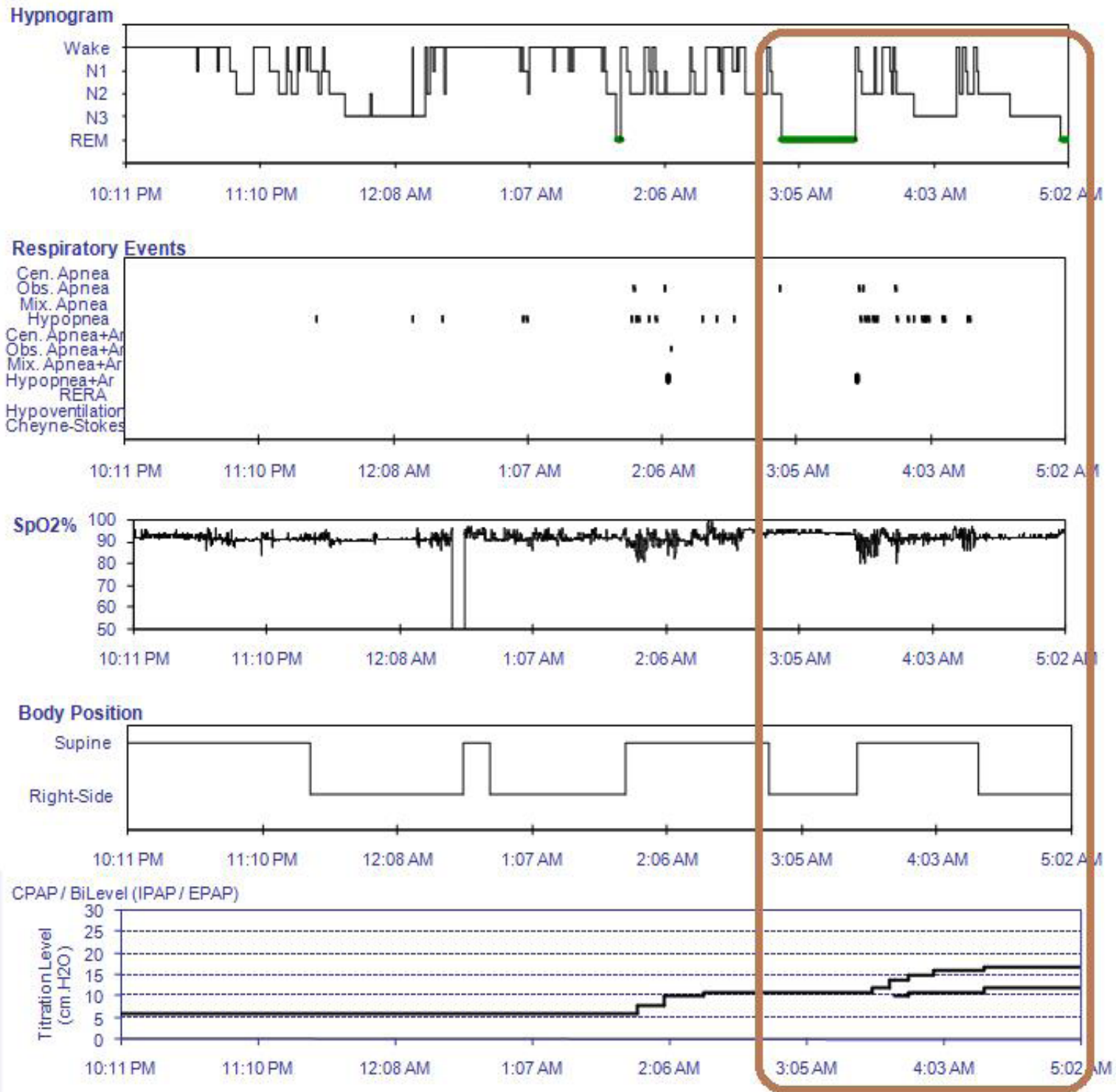

Careful examination of the region within the brown rectangle in the above figure illustrates that body position is more important than the PAP level. Home sleep tests do not record body position to keep costs low. Auto-titrating CPAP devices also do not account for body position. This patient's problem would go unrecognized in a world where laboratory sleep tests are denied. While in many patients the added information of a laboratory study is not worth the additional cost, in some patients it is. Blanket denial or blanket requirement of laboratory sleep tests will not work as well as the freedom to choose what type of test is required. With freedom come responsibility and the freedom to choose works only when the costs of choices are accepted by the person receiving the benefit. Third party payers do not have the same goals as patients. Absent government regulation, the choice of sleep testing would be no more of a problem than choosing what one eats for the next meal. Some people would prefer high cost high quality options, and others would prefer low cost low quality options. 
Sleep specialists may have carved out a monopoly privilege to perform an ever declining service. It is not inconceivable that Home Sleep Tests might be offered for free by Durable Medical Equipment (DME) suppliers to capture profits in CPAP machines much as DME suppliers will perform overnight oximetry at nominal charges in order to capture prescriptions for home oxygen. The future of sleep medicine rests largely with the whims of the Centers for Medicare and Medicaid Services (CMS) bureaucracy. There will always be a small group of patients that cannot be treated with protocols designed for the "average" case. The irony of protocol driven care is that to the extent that assembly line protocols cover the population, there is no demand for the expertise of trained professionals.

Author Affiliation: Dr. Gilbert Berdine is a pulmonary physician in the Department of Internal Medicine, TTUHSC.

Received: $6 / 22 / 2016$

Accepted: $7 / 1 / 2016$

Published electronically: 7/15/2016
5. Reversal of obstructive sleep apnoea by continuous positive airway pressure applied through the nares.

http://www.ncbi.nlm.nih.gov/pubmed/6112294/

http://ac.els-cdn.com/S0140673681921401/1-s2.0S0140673681921401-main.pdf? tid $=0019857 \mathrm{e}-2 \mathrm{cc} 9-11 \mathrm{e} 6-\mathrm{a} 682$ -00000 aab0f01\&acdnat $=14653 \overline{15} 428 \_58$ ceceb0f798cd803a4bfe81ae7fb302

Lancet 1981 April 18; 1(8225): 862-5.

6. OIG Report

http://oig.hhs.gov/oei/reports/oei-05-12-00340.pdf

7. Sleepless in America

http://www.thefiscaltimes.com/Articles/2012/07/23/Sleeplessin-America-A-32-4-Billion-Business

8. Genesis web page

http://www.genesishealth.com/care-treatment/neuroscience/ sleep/patient-resources/medicare-guidelines-for-cpap/

Accessed 06/20/2016 at 10::11 AM CST. 1 page long.

9. United Health Care document of lab sleep testing

https://www.unitedhealthcareonline.com/ccmcontent/ProviderII/ UHC/en-US/Assets/ProviderStaticFiles/ProviderStaticFilesPdf/ Tools \%20and\%20Resources/Policies\%20and\%20Protocols/ Medical\%20Policies/Medical\%20Policies/Attended_Polysomnography Eval Sleep Disorders.pdf

Attended polysomnography for evaluation of sleep disorders, Policy Number: 2016T0334V April 1, 2016

\section{References}

1. The Pickwick Papers. Chapter 4

2. Extreme obesity associated with alveolar hypoventilation-A Pickwickian syndrome. http://www.amjmed.com/article/0002-9343\%2856\%2990094-8/abstract

http://www.amjmed.com/article/0002-9343\%2856\%2990094-8/ pdf Amer J Med1956; 21 (5): 811-818.

3. Polygraphic study of the episodic diurnal and nocturnal (hypnic and respiratory) manifestations of the Pickwick syndrome. http://www.sciencedirect.com/science/article/ pii/000689936690117X

http://ac.els-cdn.com/000689936690117X/1-s2.0$000689936690117 \mathrm{X}$-main.pdf? tid=9d730232-2cbf-11 e6b4e9-00000aab0f01\&acdnat $=1 \overline{465311397} 20624043930 \mathrm{~d}$ c49e20ec398b5fbb0399

Brain Research 1966; 1: 167-168.

4. Pathogenesis of upper airway occlusion during sleep. http://www.ncbi.nlm.nih.gov/pubmed/670014/ http://jap.physiology.org/content/44/6/931.full.pdf+html J Appl Physiol Respir Environ Exerc Physiol 1978; 44(6): 931-8. 